\title{
Féeries
}

Études sur le conte merveilleux, XVII $-\mathrm{XIX}{ }^{\mathrm{e}}$ siècle

\section{Seuils et continuité dans les Contes de Charles} Perrault

Thresholds and continuity in Charles Perrault's fairy tales

\section{Delphine Reguig}

\section{(2) OpenEdition}

1 Journals

Édition électronique

URL : http://journals.openedition.org/feeries/1995

DOI : $10.4000 /$ feeries. 1995

ISSN : 1957-7753

Éditeur

UGA Éditions/Université Grenoble Alpes

Édition imprimée

ISBN : 978-2-37747-075-4

ISSN : 1766-2842

\section{Référence électronique}

Delphine Reguig, "Seuils et continuité dans les Contes de Charles Perrault», Féeries [En ligne], 15 |

2018, mis en ligne le 24 janvier 2019, consulté le 08 septembre 2020. URL : http://

journals.openedition.org/feeries/1995; DOI : https://doi.org/10.4000/feeries.1995

Ce document a été généré automatiquement le 8 septembre 2020

(c) Féeries 


\section{Seuils et continuité dans les Contes de Charles Perrault}

Thresholds and continuity in Charles Perrault's fairy tales

Delphine Reguig

On n'entre pas en féerie sans franchir certaines marches, sans ouvrir certaines portes, sans dépasser certaines limites. La formule «Il était une fois » rend évident le passage auquel invite le conte de fées vers un univers autre, où le rapport aux cadres référentiels et logiques ordinaires se trouve désorienté. La richesse du frontispice gravé pour l'édition Barbin de 1697, qui retint l'attention de Louis Marin ${ }^{1}$, le souligne: le corpus offert par les Histoires ou Contes du Temps passé de Charles Perrault montre que le monde du conte de fées se présente à son lecteur avec une étrangeté assumée. La mise en scène y insiste sur la clôture de l'espace de l'énonciation conteuse et manifeste " la toute-puissance maîtresse de la voix narratrice ${ }^{2}$ ». L'image du sommeil de la raison, mobilisée dans les premiers vers de Peau d'Âne, souligne quant à elle la limite entre la vigilance et son abolition comme son effacement par le conte même chez le lecteur: « Pourquoi faut-il s'émerveiller / Que la Raison la mieux sensée, / Lasse souvent de trop veiller, / Par des contes d'Ogre et de Fée / Ingénieusement bercée, / Prenne plaisir à sommeiller ${ }^{3}$ ?" La mise en abyme de ce motif dans La Belle au bois dormant est remarquable: si, au grand réveil du château et de ses occupants, le prince est pris au dépourvu, la princesse quant à elle se montre tout à fait éloquente puisque son «si long sommeil» lui a donné «le temps de songer à ce qu'elle aurait à lui dire » en lui procurant «le plaisir des songes agréables $»^{4}$. Le personnage du conte fait là explicitement l'expérience de la traversée des états de conscience distincts. Et ce type d'éloignement est une étape fondamentale de son parcours comme elle l'est pour le lecteur.

1 Par un retour particulièrement subtil du procédé de la mise en abyme observé dans le frontispice de 1697, le conte dévoile ainsi l'un des effets de l'enchantement féerique : faire communiquer deux mondes en les séparant, faire advenir un échange entre deux configurations en soulignant néanmoins la différence entre elles. Du point de vue de la poétique du conte, ce paradoxe actif tend à neutraliser la logique binaire et le principe 
de non-contradiction alors même qu'il repose sur un usage ouvertement ludique des binarités, symétries, jeux de régularités organisant les relations entre l'espace extérieur au conte et sa fictionnalité interne, les mouvements des personnages d'un lieu à l'autre, les rapports entre les personnages eux-mêmes. Dans Les Fées par exemple, la corvée d'eau implique un éloignement « à une grande demi-lieue du logis ${ }^{5}$ » et c'est à la faveur de cet éloignement que la fille cadette rencontre la fée. L'antithèse structure alors la suite du conte au fur et à mesure que la fée distribue ses dons : " deux Roses, deux Perles, et deux gros Diamants ${ }^{6}$ " sortent de la bouche de la belle et bonne fille munie de la simple cruche tandis que « deux vipères et deux crapauds » sortent de celle de la bouche de la fille aînée, "brutale orgueilleuse ", quoique munie d'un flacon d'argent. Sans être parfaite, la symétrie inversée est signifiante: elle réfère à une logique autre, propre à l'univers du conte et capable de structurer l'ensemble par la distance que le conte ménage avec un autre monde ${ }^{7}$. La naissance informe de Riquet à la houppe appelle à nouveau la structuration, fruit de la mise en place d'une symétrie formelle dans un cadre certes fantaisiste mais soumis à l'instance médiatrice de la fée, pourvoyeuse de mesure et d'équilibre : l'enfant est laid mais plein d'esprit. Comme dans Les Fées, l'arithmétique du conte est visible sans être rigoureusement exacte: la symétrie place face à Riquet non pas une seule princesse mais deux princesses également pourvues de qualités opposées et compensant en miroir les défauts du jeune homme. La première princesse est belle mais stupide; la seconde laide mais pleine d'esprit. La trajectoire ludique du conte consiste à faire correspondre, par la magie de l'échange des dons et la traversée des apparences, les lacunes des uns aux qualités des autres. Le conte rappelle que la perfection n'est d'aucun monde et que, pour parvenir à l'unité et à la plénitude, le passage d'un monde à l'autre est profitable. La figure de l'inversion structurelle autour du pivot frontalier de la différence réapparait dans Le Petit Poucet où la symétrie entre les sept garçons égarés et les sept filles de l'Ogre permet de renverser le péril et de sauver les premiers à la faveur d'un basculement narratif radical qui décloisonne les univers initialement étanches.

2 Pour permettre ces échanges et ces renversements entre les situations et les données narratives, les figures du seuil font l'objet de toute l'attention du conteur: portes et heures, frontières physiques ou temporelles, limites spatiales et chronologiques, traits innés et déterminations naturelles assurent à la fois la liberté et la cohérence fictionnelles du conte. Univers autonome et cloisonné, le genre, chez Perrault, tend ainsi à élaborer le fait même du passage pour en faire un motif littéraire. Le conte ne se contente pas de signifier au lecteur une frontière entre son univers de référence et l'univers féerique. Le genre incite son lecteur à prêter une attention particulière aux passages entre espaces internes disjoints et à la manière dont le récit dote ces effets d'une valeur dramatique et symbolique. Cette organisation du conte de fées autour de jalons saillants du point de vue spatial et temporel est aussi une manière de faire écho à la distance que le genre ménage avec le régime ordinaire de la lecture. Le conte, précisément parce qu'il institue un univers parallèle, appelle la constitution d'une nouvelle régulation pour créer les conditions d'une motivation interne assumant un arbitraire choisi : parce que son franchissement est l'occasion d'effets de rites marqués, le seuil devient ainsi un marqueur générique pour le conte. L'objet de ces quelques réflexions est d'examiner l'enrichissement du motif du seuil dans le processus de symbolisation propre au conte: susceptible de faire vaciller toute limitation herméneutique, ce motif semble recéler une part notable de la force dialectique de l'imaginaire féerique. 


\section{L'univers gradué des Contes}

Le monde physique des contes se présente comme un univers spatial cloisonné et gradué par les effets de seuil. Furetière définit ce terme comme « La marche, la pièce de bois ou de pierre qu'on met au bas de la porte » : on peut être arrêté par le seuil, rester sur le seuil, mais on peut aussi le passer et en faire le point de départ d'une progression. Dans les Contes, Perrault choisit de marquer les seuils. La logique narrative ne mène pas les personnages d'une situation initiale à une situation finale de manière linéaire : elle les place sur un chemin dont le parcours suppose des franchissements nécessaires; elle les conduit à réaffirmer constamment le mouvement dont ils sont acteurs en les confrontant à la possibilité de l'immobilité devant la marque du seuil. Or, en dépit de la prégnance de cette image, qui implique des ruptures, le mouvement narratif ne souffre pas de discontinuité, bien au contraire. Le déroulement logique et chronologique du conte se présente comme un itinéraire orienté dans l'espace et dans le temps: le personnage progresse et son avancée est scandée par des passages. Ces derniers se trouvent figurés selon trois modalités parentes, et non exclusives, selon le degré de sanction narrative que le conteur choisit de souligner: soit le seuil est fermement incarné dans le motif de la "porte" et il invite à un mouvement qui inclut le dépassement; soit il se reconnaît à une corrélation spatio-temporelle qui lie basculement chronologique et rupture spatiale, et l'heure décide d'un déplacement, voire d'une métamorphose; soit il tend à s'effacer dans un continuum spatial dynamique où, moins sensible, il n'est qu'implicitement présent. Dans ce dernier cas, le motif du seuil se trouve absorbé dans une catégorie imprécise, le «lointain », espace confus où s'enfoncent les personnages et dont l'indétermination renforce encore la distance. Dans tous les cas, le passage de seuils s'impose aux personnages comme une condition de leur insertion dans la continuité narrative et de leur appartenance à la logique du conte.

3 Dans Griselidis, l'image est spontanément propice à une contamination du concret par l'abstrait: le travail narratif sur le mouvement dans l'espace extérieur nourrit la figuration polysémique de l'espace intérieur. Au début du conte, le Prince part à la chasse avec ses compagnons mais ne tarde pas à s'éloigner en prenant "une route détournée » qui le désolidarise du groupe initial : «Plus il court, plus il s'en sépare ${ }^{8}$ ». Ce départ l'égare pour qu'il trouve la jeune fille correspondant aux attentes qu'il a formulées quelques vers plus haut. Or la jeune fille se présente comme capable de remettre les pas du prince «sur un chemin connu » et de lui faire quitter son choix de solitude. L'interprétation est d'autant plus orientée que le terme de destinée vient se surimposer à celui de «chemin " pour indiquer un mouvement lui-même vectorisé. Au retour, le conteur mentionne que le Prince cartographie l'espace qui sépare la Ville de l'espace où lui est apparue la bergère ${ }^{9}$. Il prépare ainsi la possibilité d'un retour, d'une circulation entre des lieux séparés par le seuil de la forêt. Cette circonvolution géographique métaphorise le parcours affectif et mental du prince que le fantasme de transparence absolue pourrait condamner à la rigidité. Le retour s'effectue effectivement «malgré les traverses / De cent routes diverses ${ }^{10}$ » dont les seuils s'effacent devant l'amour qui le guide. Le parcours narratif implique le passage d'un lieu à un autre mais surtout le franchissement de frontières, naturelles ou matérielles, qui séparent d'abord le personnage d'une nouveauté à rencontrer. 
Car si le cloisonnement des espaces est symbolique d'une séparation des natures et des milieux, la mention du franchissement de seuils par les personnages ménage des effets de clôture et d'ouverture entre ces espaces, effets qui se trouvent investis d'un rôle structurant dans la succession dramatique des épisodes du conte. Ainsi, le départ des sœurs de Cendrillon au bal sert-il de clausule à un premier moment du conte, avant que la narration ne prenne un tour plus favorable pour le personnage principal: «Enfin l'heureux jour arriva, on partit, et Cendrillon les suivit des yeux le plus longtemps qu'elle put ; lorsqu'elle ne les vit plus, elle se mit à pleurer ${ }^{11}$. » Espace fictionnel et unité narrative semblent ainsi correspondre et se construire parallèlement en se conformant aux mêmes limites. Dans Peau d'Âne, pour fuir un père déviant et obstiné, la princesse, dit le conteur, recule les profondeurs de l'espace : «Elle alla donc bien loin, bien loin, encor plus $\operatorname{loin}^{12}$;", avec une répétition qui vient à bout des seuils qui pourraient arrêter le personnage. La princesse se trouve alors préservée à l'abri d'un "huis clos » spatio-temporel intime où, le dimanche, elle se pare des robes magiques dans lesquelles «Elle aimait à se voir jeune, vermeille et blanche / Et plus brave cent fois que nulle autre n'était ; / Ce doux plaisir la sustentait / Et la menait jusqu'à l'autre Dimanche ${ }^{13}$ ". La densité existentielle de cet espace-temps préservé est clairement liée à la possibilité de passages entre des apparences différentes. C'est pourquoi, dans la scénographie qui organise les données fondamentales du récit, le motif du seuil possède une valeur dramatique et poétique à la fois. Lorsque le prince, «errant à l'aventure » découvre Peau d'Âne, «Par hasard il m[e]t l'œil au trou de la serrure ${ }^{14} »$. Cette absence de motivation affichée met en valeur la réaction émotionnelle du prince, si «touché » et "comblé de plaisir» par la contemplation de la princesse, que "Trois fois, dans la chaleur du feu qui le transporte, / Il voulut enfoncer la porte; / Mais croyant voir une Divinité, / Trois fois par le respect son bras fut arrêté. $»^{15}$ La répétition sert $l^{\prime} h y p e r b o l{ }^{16}$; elle fait aussi écho au triple mouvement d'éloignement de la princesse « bien loin, bien loin, encor plus loin » : le prince rejoint Peau d'Âne vers la profondeur de l'univers féerique.

5 La sanction du seuil est parfois inquiétante: pénétrer l'espace étranger expose au risque. La porte de la maison de la grand-mère du Petit Chaperon rouge ne s'ouvre pas sur la sécurité ${ }^{17}$, et celle que l'épouse de Barbe bleue hésite à ouvrir figure une transgression irrémédiable ${ }^{18}$. Il faut néanmoins passer des seuils pour faire avancer le conte et la Barbe bleue l'apprend à ses dépens puisque le retour à l'ordre est obtenu à la faveur de l'intervention, par la porte («Dans ce moment on heurta si fort à la porte, que la Barbe bleue s'arrêta tout court»), des proches qui viennent au secours de son épouse condamnée. Perrault joue de cette superposition entre franchissement des seuils spatiaux-temporels et revirements narratifs, ménageant ainsi des effets de contrastes d'autant plus saillants. La porte hiérarchise, sépare, distingue, mais permet aussi de réunir, une fois le seuil franchi. Lorsque le petit Poucet retrouve le chemin de sa maison au moyen de son ingénieux balisage et y reconduit ses frères " par le même chemin qu'ils étaient venus dans la forêt. Ils n'osèrent d'abord entrer, mais ils se mirent tous contre la porte pour écouter ce que disaient leur Père et leur Mère ${ }^{19} »$ :

La Bûcheronne était toute en pleurs. " Hélas ! où sont maintenant mes enfants, mes pauvres enfants? » Elle le dit une fois si haut que les enfants qui étaient à la porte, l'ayant entendu, se mirent à crier tous ensemble : « Nous voilà, nous voilà. » Elle courut vite leur ouvrir la porte, et leur dit en les embrassant: «Que je suis aise de vous revoir, mes chers enfants ${ }^{20}$ ! 
La loi du conte agit néanmoins encore : il faut franchir à nouveau la porte, s'éloigner, "mener [les enfants] bien plus loin que la première fois" et élaborer le projet "secrètement ", c'est-à-dire en rendant la séparation imperméable, en empêchant le trop malin petit Poucet de sortir rassembler les cailloux nécessaires à son orientation dans l'espace : « [...] il trouva la porte de la maison fermée à double tour. $»^{21}$ Une fois livrés à l'espace magique de la forêt, les enfants repèrent une chandelle qui les guide vers un nouveau seuil : «Ils heurtèrent à la porte, et une bonne femme vint leur ouvrir $^{22}$. " La maison est inhospitalière, mais moins que la forêt nocturne. À peine la retraite élue par nécessité, le conte rappelle l'étrangeté de l'espace investi en mentionnant le franchissement de son seuil: «[...] ils entendirent heurter trois ou quatre grands coups à la porte : c'était l'ogre qui revenait. » Dans l'univers féerique, il faut qu'une porte s'ouvre et se ferme, que les espaces communiquent, que les mondes échangent, pour que le conte puisse avancer et la lecture s'approfondir. Le motif de la forêt figure l'extrême de la profondeur symbolique de l'espace ouvert par le conte, une fois passées les portes les plus familières. Il combine les traits de l'éloignement et du cloisonnement compris dans l'image du seuil. C'est dans la forêt qu'a lieu la rencontre entre Riquet à la houppe et son double inversé, la princesse qui « eût donné sans regret toute sa beauté pour avoir la moitié de l'esprit de sa sœur ${ }^{23} »$ :

Un jour qu'elle s'était retirée dans un bois pour y plaindre son malheur, elle vit venir à elle un petit homme fort laid et fort désagréable, mais vêtu très magnifiquement. C'était le jeune Prince Riquet à la houppe, qui étant devenu amoureux d'elle sur ses Portraits qui couraient par tout le monde, avait quitté le Royaume de son père pour avoir le plaisir de la voir et de lui parler. Ravi de la rencontrer ainsi toute seule, il l'aborde avec tout le respect et toute la politesse imaginable ${ }^{24}$.

Les éléments déjà relevés viennent structurer la scène: l'éloignement des deux personnages par rapport à leur univers de référence ; l'antithèse qui les rapporte l'un à l'autre de part et d'autre d'une séparation ; l'hyperbole qui rend possible l'« abord ». Lié à la figuration des obstacles, de leur affrontement et de leur dépassement, le motif du seuil, ainsi délibérément épuré par la logique féerique, organise et soutient la dynamique du récit.

\section{Passer le seuil}

L'insistance de la narration sur la figure du seuil et sur les effets liés au franchissement de seuils, dans la progression dramatique, confère au motif une valeur logique. De part et d'autre du seuil, les contraires s'articulent avec l'énergie du paradoxe. C'est le constat que fait le prince découvrant Peau d'Âne derrière sa porte : « Il s'enquit quelle était cette Nymphe admirable / Qui demeurait dans une basse-cour, / Au fond d'une allée effroyable, / Où l'on ne voit goutte en plein jour ${ }^{25}$. » Reléguer Cendrillon « au coin de la cheminée ", à la transition entre l'espace du partage familial et celui du retrait isolé, c'est aussi justifier l'hyperbole qui compense en valeur symbolique le phénomène d'éloignement physique :

Lorsqu'elle avait fait son ouvrage, elle s'allait mettre au coin de la cheminée, et s'asseoir dans les cendres, ce qui faisait qu'on l'appelait communément dans le logis Cucendron. La cadette, qui n'était pas si malhonnête que son aînée, l'appelait Cendrillon; cependant Cendrillon, avec ses méchants habits, ne laissait pas d'être cent fois plus belle que ses sœurs, quoique vêtues très magnifiquement ${ }^{26}$.

En ménageant des degrés dans le conte, par l'usage réitéré et affiché du procédé du palier narratif et spatial, le conteur attire l'attention sur la relation étroite qui existe 
entre le genre du conte et la transgression: plus la progression féerique traverse de seuils, plus cette avancée se conquiert comme un excès qu'on ne saurait circonscrire. L'apparence immonde de Peau d'Âne avant sa porte précipite l'appréciation du personnage dans le dégoût superlativement exprimé, mais ce n'est qu'une version négative de la disproportion à laquelle la féerie incline naturellement :

Cette Peau d'Âne est une noire Taupe

Plus vilaine encore et plus gaupe

Que le plus sale Marmiton.

- N'importe, dit la Reine, il le faut satisfaire

Et c'est à cela seul que nous devons songer.

Il aurait eu de l'or, tant l'aimait cette Mère,

$S$ 'il en avait voulu manger ${ }^{27}$.

La course des enfants fuyant l'Ogre dans Le Petit Poucet dure "presque toute la nuit, toujours en tremblant et sans savoir où ils allaient ", et il faut bien des bottes de sept lieux à leur poursuivant pour aller «de montagne en montagne » et traverser "des rivières aussi aisément qu'il aurait fait le moindre ruisseau $»^{28}$. La récurrence du motif du seuil questionne et relativise les proportions et les mesures. Il fait du conte le genre où il s'agit de passer ou de ne pas passer, traverser ou rester entravé : en situation, la bague de Peau d'Âne ou la pantoufle de Cendrillon posent le problème en termes très concrets. La première « toujours trop petite ou trop ronde / D'un dédain presque égal rebutait tout le monde » : il faut que l'héroïne, maîtresse des seuils, des représentations contradictoires et des logiques paradoxales, tire « de dessous sa peau noire / Une petite main qui semblait de l'ivoire" pour que «de la Bague fatale,/ D'une justesse sans égale/ Son petit doigt [soit] entouré $»^{29}$. La réaction du public doit inspirer celle des lecteurs absorbés dans l'imaginaire illimité du conte par la vertu même des limites marquées : «La Cour fut dans une surprise / Qui ne peut pas être comprise. » Comme la clé, qui ouvre la porte, les bottes sont magiques et s'ajustent, se déforment au gré des espaces traversés par les personnages :

Le petit Poucet s'étant approché de l'Ogre, lui tira doucement ses bottes, et les mit aussitôt. Les bottes étaient fort grandes et fort larges; mais comme elles étaient Fées, elles avaient le don de s'agrandir et de s'apetisser selon la jambe de celui qui les chaussait, de sorte qu'elles se trouvèrent aussi justes à ses pieds et à ses jambes que si elles avaient été faites pour lui ${ }^{30}$.

Une telle mobilité s'illustre encore dans l'adaptation à l'échelle sociale qu'elle autorise à certains personnages comme le chat botté. Ce dernier commence par franchir des seuils physiques et sa démarche se double rapidement d'une progression sociale : «[...] il s'en alla chez le Roi et demanda à lui parler. On le fit monter à l'Appartement de sa Majesté, où étant entré il fit une grande révérence au Roi, et lui dit [...] ${ }^{31}$.» Le renversement de situation qui advient à l'animal, avant d'advenir à son maître, est matérialisé par un changement de lieu et de configuration relationnelle: «Le Roi voulut qu'il montât dans son Carrosse, et qu'il fût de la promenade ${ }^{32}$. » Pour " arriver ", le Chat ne cesse d'arriver dans de nouveaux lieux (" Le maître Chat arriva enfin dans un beau Château dont le Maître était un Ogre, le plus riche qu'on ait jamais vu [... $\left.]^{33} »\right)$ et c'est par la même vertu du seuil, emblème de la mobilité transgressive, que le chat mange l'ogre transformé en souris. Une fois ces étapes traversées et ces espaces parcourus, le chat se fixe et c'est alors que le mouvement centripète de la féerie attire à lui le Roi «qui vit en passant le beau Château de l'Ogre » et "voulut entrer dedans " La rapidité magique contamine alors le rythme de la transformation sociale : le fils $\mathrm{du}$ meunier est invité à devenir le gendre du roi tandis que «Le Chat devint grand Seigneur, et ne courut plus après les souris, que pour se divertir ${ }^{35}$ ». Le conte s'achève 
avec la fin du cycle des traversées et des passages: on ne saurait mieux dire que l'immobilité n'a rien de féerique et que l'espace magique se traverse nécessairement.

7 Le processus sociologique est similaire dans Les Fées où le « fils du Roi » trouve la jeune fille vertueuse " toute seule ", " chassée du logis " par sa mère avant d'être converti à la magie de sa bouche parlant perles et diamants et de l'emmener au Palais du Roi son père où il l'épouse. Cette fois la symétrie agit cruellement contre la sœur, à son tour chassée par la mère mais dont la course échoue à «trouver personne qui voulût la recevoir " avant de la conduire à aller "mourir au coin d'un bois " ${ }^{36}$. Là encore, les derniers mots du conte voient s'exténuer la féerie avec le mouvement, comme si le conte ne pouvait survivre à l'inertie et se nourrissait de surenchères dans les transitions. Cette logique de la surenchère est particulièrement évidente dans Cendrillon : l'hérö̈ne, remarquée lors du bal où elle se rend clandestinement, est installée par le fils du roi "à la place la plus honorable », avant d'être choisie comme partenaire dans la danse; après ce premier palier, il ne s'agit pas de s'arrêter là : "Dès qu'elle fut arrivée, elle alla trouver sa Marraine, et après l'avoir remerciée, elle lui dit qu'elle souhaiterait bien aller encore le lendemain au Bal, parce que le Fils du Roi l'en avait priée. » La gradation spatiale et sociale est enclenchée pour organiser la progression narrative: "Le lendemain les deux sœurs furent au Bal, et Cendrillon aussi, mais encore plus parée que la première fois. $\${ }^{37}$ Entrer et sortir, pénétrer des mondes différents sont des impératifs pour les personnages dont la mobilité symbolique organise la temporalité féerique et recoupe des enjeux imaginaires, sociaux et politiques. Certes le conte, notamment par la brièveté et la condensation poétique qui le caractérisent, se présente nécessairement comme un genre de l'adaptabilité. Mais l'usage métapoétique que Perrault fait de ces ruptures permet au passage par le seuil de déterminer la réalisation de la métamorphose féerique.

\section{Seuils et métamorphoses}

En matérialisant régulièrement le seuil entre la fiction et l'adresse directe au lecteur, Perrault fait enfin de l'image un outil ouvertement poétique, un marqueur générique visible. On citera dans ce contexte le passage de Peau d'Âne où le conteur intervient pour contester l'interprétation magique de la contemplation fortuite de la princesse par le prince, et la concurrencer par une interprétation factuelle et prosaïque : la frontière de la porte n'aurait été qu'artificiellement maintenue par la princesse qui aurait perçu la présence du prince et aurait délibérément fait choir son anneau dans la pâte du gâteau qu'elle confectionnait ${ }^{38}$. De fait, plus le seuil s'instaure entre le vraisemblable et le magique, plus il met en valeur la liberté du conteur qui emploie son

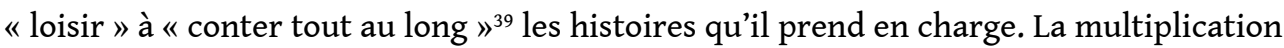
des seuils détermine en quelque sorte, avec le destin du personnage, l'extension maximale de la parole conteuse ${ }^{40}$. Conter tout le conte, assumer la traversée du réel, c'est autoriser les fins matrimoniales triomphales où la parole magique est mise en abyme : « Dans ce moment la Marraine arriva / Qui raconta toute l'histoire, / Et par son récit acheva / De combler Peau d'Âne de gloire ${ }^{41}$. » Il n'est plus question de mettre en doute la vraisemblance de la narration : sa mise en crise est largement compensée par la force de la cohésion interne acquise par le conte: "Le Conte de Peau d'Âne est difficile à croire, / Mais tant que dans le Monde on aura des Enfants, / Des Mères et des Mères-grands, / On en gardera la mémoire ${ }^{42}$. " Le conte invite son lecteur à rejoindre un espace magique qui tend à se décloisonner. 
La désinvolture du conteur, exemplaire dans La Belle au bois dormant, peut rendre ce lecteur complice d'une narration aussi rapide que légère. La motivation des faits qui forment la trame narrative fait alors l'objet d'un jeu où la féerie charme d'autant plus qu'elle paraît artificielle ( Tout cela se fit en un moment; les Fées n'étaient pas longues à leur besogne $\left.{ }^{43} »\right)$. L'humour rend l'avancée du conte aussi gratuite à l'égard d'une logique ordinaire que nécessaire à l'égard de la logique féerique : « Il ne laissa pas de continuer son chemin : un Prince jeune et amoureux est toujours vaillant ${ }^{44}$.» C'est alors un lecteur lui-même d'autant plus mobile qui admire la manière dont les fées "font aller»-comme la Marraine de Cendrillon-, les héros vers leur accomplissement :

Tu voudrais bien aller au Bal, n'est-ce pas? Hélas oui, dit Cendrillon en soupirant. Hé bien, seras-tu bonne fille? dit sa Marraine, je t'y ferai aller. Elle la mena dans sa chambre, et lui dit : Va dans le jardin et apporte-moi une citrouille. Cendrillon alla aussitôt cueillir la plus belle qu'elle put trouver, et la porta à sa Marraine, ne pouvant deviner comment cette citrouille la pourrait faire aller au bal ${ }^{45}$.

Pour « deviner », il faut passer par le rituel du seuil : sortir, aller, rentrer, et enfin vivre la métamorphose. La répétition des formules de la Marraine ( Va dans le jardin, tu y trouveras six lézards, derrière l'arrosoir, apporte-les $\mathrm{moi}^{46} »$ ) est éloquente. Elle crée un mouvement de passage régulier dont la récurrence approfondit la signification symbolique. La traversée féerique rend Cendrillon méconnaissable au sens propre: » Elle alla s'asseoir auprès de ses sœurs, et leur fit mille honnêtetés : elle leur fit part des oranges et des citrons que le Prince lui avait donnés, ce qui les étonna fort, car elles ne la connaissaient point ${ }^{47}$. " Le miroir sans tain derrière lequel la frontière magique place le personnage agit comme une cloison unilatérale entre ceux que le conte rend mobile et ceux qu'il immobilise. «La plus belle Princesse, la plus belle qu'on puisse jamais voir ${ }^{48}$ ", ne peut être nommée: son identité échappe, protégée par l'invraisemblance magique. C'est pourquoi le seuil chronologique possède la valeur d'un impératif : il ne faut pas "passer minuit ", il ne faut pas se soustraire à la loi du seuil sous peine de voir la métamorphose s'inverser et la magie se résorber en rigidité close.

9 Le dispositif sur lequel est construit Cendrillon est exemplaire. Son organisation contribue à rattacher la poétique du seuil à l'exploration de la question de l'identité : derrière la porte et la clé, après le passage, c'est à l'altérité que l'on a affaire. Le miroitement des apparences que permet la frontière horaire fait hésiter la lecture : Cendrillon est-elle elle-même avant ou après minuit? Le lecteur reconnaît bien le personnage de part et d'autre de la limite mais c'est le franchissement de la limite qui la révèle précisément à elle et aux autres :

Le Fils du Roi fut toujours auprès d'elle, et ne cessa de lui conter des douceurs; la jeune Demoiselle ne s'ennuyait point, et oublia ce que sa Marraine lui avait recommandé, de sorte qu'elle entendit sonner le premier coup de minuit, lorsqu'elle ne croyait pas qu'il fût encore onze heures : elle se leva et s'enfuit aussi légèrement qu'aurait fait une biche : le Prince la suivit, mais il ne put l'attraper; elle laissa tomber une de ses pantoufles de verre, que le Prince ramassa bien soigneusement. Cendrillon arriva chez elle bien essoufflée, sans carrosse, sans laquais, et avec ses méchants habits, rien ne lui étant resté de toute sa magnificence qu'une de ses petites pantoufles, la pareille de celle qu'elle avait laissé tomber. On demanda aux Gardes de la porte du Palais s'ils n'avaient point vu sortir une Princesse ; ils dirent qu'ils n'avaient vu sortir personne, qu'une jeune fille fort mal vêtue, et qui avait plus l'air d'une Paysanne que d'une Demoiselle ${ }^{49}$. 
L'impulsion du seuil provoque un mouvement où le personnage se transforme à nouveau et cette fois en un être de transition, une créature dont la comparaison animale du personnage avec une biche souligne l'hybridité. L'enchaînement reconduit Cendrillon «chez elle » dans un espace familier qui rappelle que l'énergie féerique l'a transportée dans un univers étranger. Mais du "rien» que laisse la résorption magique, il reste un petit miracle mi-féerique, mi-ordinaire grâce auquel Perrault, soulignant le raccord entre les deux régimes d'écriture, joue encore avec le seuil de l'invraisemblance conteuse. La séparation de la paire de pantoufles est permise par le renouvellement du jeu constant avec la symétrie : l'objet, distinct d'une part et d'autre des espaces en jeu, devient lui-même emblématique du potentiel magique du seuil. Ce dernier, malgré les gardes qui pourraient interdire tout passage par les portes du palais, rend toujours possible la réunion des deux Cendrillon, celle d'avant minuit et celle d'après la magie. Faire coïncider les deux personnages, les deux pantoufles, réunir la paire, réunir la bonté et la beauté, telle est l'opération que promet le seuil. L'ajustement parfait conduit à un effacement du seuil sur lequel se clôt la résolution du conte. Le traitement de la question de l'identité demande une stabilisation à partir de signes de reconnaissance qui déterminent le renversement narratif ${ }^{50}$ et dont la médiation rend inutile la dynamique du seuil. Pour retrouver Cendrillon, l'impératif de la coïncidence est publié «à son de trompe»: «[...] il épouserait celle dont le pied serait bien juste à la pantoufle ${ }^{51}$. On connaît l'échec des essayages répétés, jusqu'aux sœurs de Cendrillon. Nous remarquerons surtout que Cendrillon est elle-même l'agent de la coïncidence : «Cendrillon qui les regardait, et qui reconnut sa pantoufle, dit en riant : que je voie si elle ne me serait pas bonne, ses sœurs se mirent à rire et à se moquer d'elle ${ }^{52}$.» Contre la raillerie, la vraisemblance de l'improbable proposition s'impose au gentilhomme qui regarde " attentivement Cendrillon, et la trouvant fort belle, dit que cela était juste ». Cendrillon est donc pertinente, puis « juste comme de cire » dans la pantoufle. Comme ailleurs dans les Contes, ce moment d'effacement du seuil provoque «l'étonnement ». Ce dernier est ici majoré par la plume de Perrault qui présente la réunion des univers précédemment scindés à nouveau sur le mode de la surenchère : "L'étonnement des deux sœurs fut grand, mais plus grand encore quand Cendrillon tira de sa poche l'autre petite pantoufle qu'elle mit à son pied $^{53}$.» L'intervention de la Marraine confirme l'inutilité désormais de la mobilité magique promise par la frontière puisque l'hyperbole conjoint à présent l'ordinaire et la féerie : "Là-dessus arriva la Marraine, qui ayant donné un coup de sa baguette sur les habits de Cendrillon, les fit devenir encore plus magnifiques que tous les autres ${ }^{54}$. $\mathrm{La}$ reconnaissance peut alors être complète : «Alors les deux sœurs la reconnurent pour la belle personne qu'elles avaient vue au $\mathrm{Bal}^{55}$. " Cendrillon retrouve Cendrillon là où sa bonté la mène et où elle invite finalement ses sœurs : « On la mena chez le jeune Prince, parée comme elle l'était : il la trouva encore plus belle que jamais, et peu de jours après, il l'épousa. Cendrillon qui était aussi bonne que belle, fit loger ses deux sœurs au Palais, et les maria dès le jour même à deux grands Seigneurs de la Cour ${ }^{56}$.»

10 Au départ pourtant, comme on l'a vu, le conte procède volontiers par défiguration première de ses personnages : "Pour vous rendre méconnaissable, / La dépouille de l'Âne est un masque admirable. / Cachez-vous bien dans cette peau, / On ne croira jamais, tant elle est effroyable, / Qu'elle renferme rien de beau ${ }^{57}$. » La logique féerique repose sur le seuil comme condition de la métamorphose de personnages d'abord privés de complétude, éloignés dans leur différence. On a déjà souligné comment, de manière emblématique, Riquet à la houppe fait du retour à l'unité le motif structurant du 
conte. Le personnage éponyme se présente à la princesse qu'il souhaite épouser comme détenteur d'un " pouvoir ", celui qui consiste à offrir l'harmonie à son interlocutrice à condition qu'un seuil chronologique soit respecté :

J'ai le pouvoir, Madame, dit Riquet à la houppe, de donner de l'esprit autant qu'on en saurait avoir à la personne que je dois aimer le plus, et comme vous êtes, Madame, cette personne, il ne tiendra qu'à vous que vous n'ayez autant d'esprit qu'on en peut avoir, pourvu que vous vouliez bien m'épouser. La Princesse demeura tout interdite, et ne répondit rien. Je vois, reprit Riquet à la houppe, que cette proposition vous fait de la peine, et je ne m'en étonne pas; mais je vous donne un an tout entier pour vous y résoudre. La Princesse avait si peu d'esprit, et en même temps une si grande envie d'en avoir, qu'elle s'imagina que la fin de cette année ne viendrait jamais ; de sorte qu'elle accepta la proposition qui lui était faite. Elle n'eut pas plus tôt promis à Riquet à la houppe qu'elle l'épouserait dans un an à pareil jour, qu'elle se sentit tout autre qu'elle n'était auparavant; elle se trouva une facilité incroyable à dire tout ce qui lui plaisait, et à le dire d'une manière fine, aisée et naturelle. Elle commença dès ce moment une conversation galante et soutenue avec Riquet à la houppe, où elle brilla d'une telle force que Riquet à la houppe crut lui avoir donné plus d'esprit qu'il ne s'en était réservé pour lui-même ${ }^{58}$.

L'humour allège à peine le propos : le même et l'autre s'échangent et se transforment ; la métamorphose atteint profondément l'être touché qui se reconnaît, comme Cendrillon, au travers du prisme de l'altérité. Le conteur retourne à la question des proportions en soulignant le déséquilibre causé par le retour de la princesse comblée : «Toute la Cour en eut une joie qui ne se peut imaginer, il n'y eut que sa cadette qui n'en fut pas bien aise, parce que n'ayant plus sur son aînée l'avantage de l'esprit, elle ne paraissait plus auprès d'elle qu'une Guenon fort désagréable ${ }^{59}$. " Mais voilà que le conte atteint le seuil chronologique prescrit au moment où la princesse franchit le seuil spatial de l'univers sylvestre : «Elle alla par hasard se promener dans le même bois où elle avait trouvé Riquet à la houppe, pour rêver plus commodément à ce qu'elle avait à faire ${ }^{60}$. » C'est alors que, très symboliquement, la terre s'ouvre sur la cuisine des futures noces de Riquet, la magie revenant expliciter le seuil du délai et son expiration par une manifestation physique spectaculaire. Le souvenir de la promesse renaît à la vue de Riquet à la houppe, qui se présente "exact à tenir [s]a parole ${ }^{61}$ " et prêt à faire coïncider les deux moments du conte comme les qualités opposées des deux personnages. Après quelques réticences initiales, l'échange des dons a lieu : Riquet peut "devenir" à son tour "le Prince du monde le plus beau et le plus aimable». L'ambiguïté délibérée des derniers mots du conteur est remarquable : il n'assure pas en effet que la métamorphose de Riquet est effective. Il écrit simplement que «La Princesse n'eut pas plus tôt prononcé ces paroles, que Riquet à la houppe parut à ses yeux l'homme du monde le plus beau, le mieux fait et le plus aimable qu'elle eût jamais $\mathrm{vu}^{62}$.» Cela suffit à accréditer la vraisemblance de la rumeur immédiatement rapportée : QQuelques-uns assurent que ce ne furent point les charmes de la Fée qui opérèrent, mais que l'amour seul fit cette Métamorphose ${ }^{63}$. " Autrement dit, chaque lecteur peut trouver dans le conte matière à réunir l'interprétation magique et l'interprétation logique, la première proposant de reconnaître la seconde dans une lecture complète car continue ${ }^{64}$.

11 Construit par degré, accentuant les transitions impossibles pour mieux valoriser les passages, ménageant d'hyperboliques paliers pour mieux souligner les franchissements, le conte de fées se tisse grâce à la figure multiple du seuil - spatial, temporel, psychologique, narratif, symbolique. La virtuosité régulière avec laquelle Perrault superpose limites et frontières confond la lecture du conte avec l'expérience 
d'une exploration sans borne des mondes imaginaire, social - mais aussi intérieur -, évoqués par le conte. Dépourvu de cette logique interne, fondée sur l'amplification et le paradoxe, le genre perdrait, avec le mécanisme de sa cohérence propre, son pouvoir sur le lecteur. Le hasard conduit les personnages devant des percées surprenantes comme l'arbitraire mène le lecteur à des enchaînements parfaitement contingents : l'image du seuil figure le régime sémantique du conte. Elle symbolise la réponse du conteur à ce que Jean-Paul Sermain appelle "l'appel du fabuleux », l'ouverture du conte "à ce qui dans l'homme vient l'arracher à son univers familier et l'emporter au-delà de lui-même dans un mouvement d'ascension ou dans un tourbillon de violences ${ }^{65}$ ». Passer le gué qui mène en féerie, c'est passer les lisières et rencontrer les différences, mêmes les plus intimes: se découvrir incomplet, inachevé, sans doute en quête. Cependant, la polymorphie du motif du seuil organise la porosité entre l'univers de référence du lecteur et celui du conte : grâce à cette continuité nouvelle, l'interprétation féerique peut incidemment être reversée à la raison du monde immédiat. École de lecture, la discontinuité féerique emmène bien loin à l'intérieur d'un récit où tous les renversements sont des approfondissements.

\section{NOTES}

1. L. Marin, «Une lisière de la lecture » [1990], repris dans Lectures traversières, Paris, Albin Michel, 1992, p. 17-25.

2. Selon l'expression de Louis Marin qui assimile l'effet de cette gravure à " une instruction liminaire, une injonction de seuil » à la lisière du conte et compare le frontispice à « une espèce de préface en image » (ibid., p. 17).

3. Ch. Perrault, Contes, éd. J.-P.Collinet, Paris, Gallimard, 1981, p. 97 . Toutes nos références renverront à cette édition notée Contes.

4. Ibid., p. 136.

5. Ibid., p. 165.

6. Ibid., p. 166.

7. Marc Fumaroli, commentant l'intertextualité de ce conte avec Les Enchantements de l'éloquence de L'Héritier, souligne " la brièveté simple et nue » du texte de Perrault qui simplifie à l'extrême les données de l'action »; au contraire, « le récit de $\mathrm{M}^{\text {lle }}$ L'Héritier enrichit ces données au point de leur donner une dimension romanesque ", «Les enchantements de l'éloquence : Les Fées de Charles Perrault ou De la littérature », Le Statut de la littérature. Mélanges offerts à Paul Bénichou, éd. M. Fumaroli, Genève, Droz, 1982, p. 164 et 172. Cette particularité stylistique contribue à faire saillir certains motifs comme celui de la symétrie inversée.

8. Ch. Perrault, Contes, p. 63.

9. «Cependant, pour trouver une route facile / Qui mène le Prince à la Ville, / Ils traversent des bois, des rochers escarpés / Et de torrents entrecoupés ; / Le Prince n'entre point dans de route nouvelle / Sans en bien observer tous les lieux d'alentour, / Et son ingénieux Amour / Qui songeait au retour, / En fit une carte fidèle. » Ibid., p. 66.

10. Ibid., p. 67.

11. Ibid., p. 172.

12. Ibid., p. 105. 
13. Ibid., p. 106.

14. Ibid., p. 107.

15. Ibid., p. 108.

16. Sur l'hyperbole dans les Contes, voir la thèse de Ch. Rousseau, Les Enchantements de l'éloquence: contes de fees et stratégies hyperboliques au XVII siecle, dir. Ch. Noille, Université Grenoble Alpes, 2013.

17. Éric Méchoulan analyse l'usage particulièrement inquiétant du motif de la porte dans Le Petit Chaperon rouge ( $\mathrm{Il}$ n'y a pas de fées, il n'y a que des interprétations : lecture du « "Petit Chaperon rouge" », PFSCL, n³7, 1992, p. 489-500.

18. Ch. Perrault, Contes, p. 151 : "Étant arrivée à la porte du cabinet, elle s'y arrêta quelque temps, songeant à la défense que son Mari lui avait faite, et considérant qu'il pourrait lui arriver qu'elle ne put la surmonter : elle prit donc la petite clef, et ouvrit en tremblant la porte du cabinet. »

19. Ibid., p. 192 : «Il se leva de bon matin, et alla au bord d'un ruisseau, où il emplit ses poches de petits cailloux blancs, et ensuite revint à la maison. On partit, et le petit Poucet ne découvrit rien de tout ce qu'il savait à ses frères. Ils allèrent dans une forêt fort épaisse, où à dix pas de distance on ne se voyait pas l'un l'autre. »

20. Ibid., p. 193.

21. Ibid., p. 194.

22. Ibid., p. 195.

23. Ibid., p. 182.

24. Ibid., p. 182-183.

25. Ibid., p. 108.

26. Ibid., p. 171-172.

27. Ibid., p. 109.

28. Ibid., p. 197-198.

29. Ibid., p. 112-113.

30. Ibid., p. 199.

31. Ibid., p. 158.

32. Ibid., p. 159.

33. Ibid., p. 160.

34. Ibid.

35. Ibid., p. 161.

36. Ibid., p. 167.

37. Ibid., p. 174-175.

38. Ibid., p. 110 : «On dit qu'en travaillant un peu trop à la hâte, / De son doigt par hasard il tomba dans la pâte / Un de ses anneaux de grand prix ; / Mais ceux qu'on tient savoir le fin de cette histoire / Assurent que par elle exprès il y fut mis ; / Et pour moi franchement je l'oserais bien croire, / Fort sûr que, quand le Prince à sa porte aborda / Et par le trou la regarda, / Elle s'en était aperçue : / Sur ce point la femme est si drue / Et son œil va si promptement / Qu'on ne peut la voir un moment / Qu'elle ne sache qu'on l'a vue./ Je suis bien sûr encor, et j'en ferais serment, / Qu'elle ne douta point que de son jeune Amant / La Bague ne fût bien reçue. »

39. Ibid., p. 97 : «Sans craindre donc qu'on me condamne / De mal employer mon loisir, / Je vais, pour contenter votre juste désir, / Vous conter tout au long l'histoire de Peau d'Âne. »

40. Voir sur ce point les réflexions nuancées de Christine Noille : «Récit en rupture, le conte se fonde aussi dans le rejet de toute évaluation qui mesurerait son rapport au monde actuel en termes d'alignement ou d'écartement plus ou moins mimétique (c'est-à-dire en termes de vérité, de vraisemblance ou de fausseté), et ce, en reprenant sur le mode ironique le postulat de la vérification confisquée, dans un énoncé désarmant de simplicité, mais logiquement constitutif : à savoir Il était une fois. S'il est vrai que les événements du conte étaient une fois, alors la voix du 
conte pose dans une assertion non sérieuse, ludique, que la vérification du conte a été opérée (ailleurs, jadis, une seule fois) dans un monde inactuel, alternatif, concurrent du monde actuel. Assertée mais non démontrée, cette vérification dans le conte ne repose pas principalement sur une contrainte logique d'écriture mais sur la seule vertu de la voix narrative introductrice, avancions-nous un peu hâtivement en la comparant aux modalités logiques de la vérification dans la nouvelle historique et galante.» («Car la clef était fée... : les raisons du récit dans les Contes de Perrault", dans Ch. Noille et B. Roukhomovsky (dir.), Un autre dix-septième siècle. Mélanges en l'honneur de Jean Serroy, Paris, Honoré Champion, 2013, p. 226.)

41. Ch. Perrault, Contes, p. 114.

42. Ibid., p. 115.

43. Ibid., p. 134. Voir aussi p. 133 : «Elle n'eut pas plus tôt pris le fuseau, que comme elle était fort vive, un peu étourdie, et que d'ailleurs l'Arrêt des Fées l'ordonnait ainsi, elle s'en perça la main, et tomba évanouie. »

44. Ibid., p. 135.

45. Ibid., p. 173.

46. Ibid.

47. Ibid., p. 174.

48. Ibid., p. 175.

49. Ibid., p. 175-176.

50. Sur l'importance de ce motif de la reconnaissance dans les Contes, voir J.-P. Sermain, Le Conte de fées du classicisme aux Lumières, Paris, Desjonquères, 2005, p. 228.

51. Ch. Perrault, Contes, p. 176.

52. Ibid.

53. Ibid.

54. Ibid., p. 176-177.

55. Ibid., p. 177.

56. Ibid.

57. Ibid., p. 104.

58. Ibid., p. 183-184.

59. Ibid., p. 184.

60. Ibid., p. 185.

61. Ibid.

62. Ibid., p. 187.

63. Ibid.

64. Pour l'analyse de ces phénomènes d'« hétérogénéité narrative " qui créent un effet de "superposition virtuose de plusieurs histoires ", voir J.-P. Sermain, ouvr. cité, p. 101 et suiv.

65. J.-P. Sermain, «Ce que les contes doivent aux fées. Liaisons anthropologiques », Féeries, $\mathrm{n}^{\circ} 7$, « Le Conte et la Fable », 2010, p. 193-201. Disponible sur http://journals.openedition.org/feeries/ 768

\section{RÉSUMÉS}

L'enchantement féerique tient largement de l'éloignement de l'univers du conte par rapport à la réalité et la logique ordinaires. Or cet éloignement n'empêche nullement de préserver la 
continuité de la narration qui fait communiquer des mondes en les séparant, fait advenir un échange entre deux univers en soulignant néanmoins la différence entre eux. Pour permettre ces échanges et ces renversements entre les situations et les données narratives, les figures du seuil font l'objet de toute l'attention du conteur: portes et heures, frontières physiques ou temporelles, limites spatiales et chronologiques, traits innés et déterminations naturelles assurent à la fois la liberté et la cohérence fictionnelles du conte. Univers autonome et cloisonné, le genre, chez Perrault, tend ainsi à élaborer le fait même du passage pour en faire un motif littéraire à valeur dramatique, logique et symbolique.

Magical spell arises from distance between fairy tale world and ordinary reality and logic. However this distance doesn't keep from ensuring the continuity of narration. In tales different worlds communicate by being splitted up. In order to allow such exchanges and inversions between narrative situations, Perrault gives his attention to threshold figures: doors and hours, spatial and chronological limits, innate characteristics and natural features provide both liberty and coherence of the tale. Autonomous and partitioned universe, fairy tale, by Perrault, therefore tends to use crossings as literary patterns with dramatic, logical, and symbolical significance.

\section{INDEX}

Mots-clés : Perrault, conte de fées, narration, identité, différence, frontières, logique, vraisemblance

Keywords : Perrault, fairy-tale, storytelling, identity, difference, borders, logic, likelihood

\section{AUTEUR \\ DELPHINE REGUIG}

Université de Lyon / Université Jean Monnet - Saint-Étienne 